\title{
A Statistical Model for Uplink Intercell Interference with Power Adaptation and Greedy Scheduling
}

\author{
Hina Tabassum\#, Ferkan Yilmaz ${ }^{\#}$, Zaher Dawy*, and Mohamed Slim Alouini\# \\ \# Electrical Engineering Program, King Abdullah University of Science and Technology (KAUST) \\ Thuwal, Mekkah Province, Saudi Arabia \\ ${ }^{*}$ Department of Electrical and Computer Engineering, American University of Beirut (AUB) \\ Beirut, Lebanon \\ \{hina.tabassum, ferkan.yilmaz, slim.alouini\}@kaust.edu.sa, zaher.dawy@aub.edu.lb
}

\begin{abstract}
This paper deals with the statistical modeling of uplink inter-cell interference (ICI) considering greedy scheduling with power adaptation based on channel conditions. The derived model is implicitly generalized for any kind of shadowing and fading environments. More precisely, we develop a generic model for the distribution of ICI based on the locations of the allocated users and their transmit powers. The derived model is utilized to evaluate important network performance metrics such as ergodic capacity, average fairness and average power preservation numerically. Monte-Carlo simulation details are included to support the analysis and show the accuracy of the derived expressions. In parallel to the literature, we show that greedy scheduling with power adaptation reduces the ICI, average power consumption of users, and enhances the average fairness among users, compared to the case without power adaptation.
\end{abstract}

\section{INTRODUCTION}

Energy efficient wireless communications has been gaining considerable attention these days mainly due to two major reasons i) dramatically varying global climate [1], and ii) slowly progressing battery technology [2]. In this context, power adaptation has been evolved as an efficient approach to reduce per capita power consumption, control inter-cell interference (ICI) and increase fairness among users in future generation wireless networks such as Orthogonal Frequency Division Multiple Access (OFDMA). In OFDMA networks, the system bandwidth is decomposed into orthogonal subcarriers. These subcarriers are adaptively allocated among users within a cell based on a predefined scheduling scheme and user transmit power levels. The allocated users on the same subcarrier in neighboring cells can cause significant uplink ICI depending on their transmit power level and channel conditions with respect to the base station (BS) of interest.

Most of the recent literature considered the modeling of ICI in the downlink where the location of interferers is usually deterministic [3], [4]. However, compared to the downlink, the modeling of ICI in the uplink is more challenging due to the arbitrary locations of the interferers and the powers associated with them. Some interesting analytical models for uplink ICI have been presented in [5], [6]; however, none of them considered the impact of channel based scheduling and power adaptation on the uplink ICI. Recently, in [7], we presented a semi-analytical framework to derive the distribution of uplink ICI on a given subcarrier assuming greedy scheduling without power adaptation. In this paper, we generalize the developed semi-analytical framework to incorporate the impact of power adaptation on the uplink ICI. This power adaptation promises considerable power savings while allowing high degree of fairness among users. Several power adaptation mechanisms are discussed in [8] such as fast and slow power control, openloop and closed-loop power control etc. In this paper we focus on slow power control considering that each user is capable of adapting its transmit power autonomously either by measuring its location through a global positioning system (GPS) or estimating its distance based on the power measurement of pilot signals from the surrounding BSs [9].

The remainder of this paper is organized as follows. Section II describes the system model. In Section III, we determine the distribution of the locations of the allocated user in a given cell. Based on this, in Section IV, we determine the distribution and moment generating function (MGF) of the ICI. In Section V, we compute important network performance metrics based on the derived MGF. Section VI presents selected numerical and simulation results. Finally, Section VII concludes the paper.

Notation: Throughout the paper, $\operatorname{Gamma}\left(m_{s}, m_{c}\right)$ denotes a Gamma distribution with shape parameter $m_{s}$ and scale parameter $m_{c} . \Gamma(\cdot)$ represents the Gamma function. $p(A)$ denotes the probability of event A. $f(\cdot)$ and $F(\cdot)$ denote the probability distribution function and cumulative distribution function, respectively. $[a, b]$ denotes a discrete set of elements which ranges from $a$ to $b$. Finally, $\mathbb{E}[\cdot]$ denotes the expectation.

\section{SySTEM MODEL}

We consider a given cell surrounded by $L$ interfering cells. For analytical convenience, the cells are assumed to be circular with radius $R$. Each cell contains $U$ uniformly distributed users where each user is assumed to have perfect knowledge of its distance to the serving BS. The rate adaptation and allocation of users on a given subcarrier, therefore, depend on the channel qualities as well as the transmit powers of the users. The instantaneous SNR of any user can then be written as:

$$
\gamma=\frac{\min \left(P_{\max }, P_{0} r^{\beta}\right) r^{-\beta} \zeta}{\sigma^{2}}
$$

where $P_{\max }[\mathrm{W}]$ is the maximum transmit power of a user, $P_{0}[\mathrm{~W}]$ is the desired power level at the receiver, $r[\mathrm{~m}]$ is the user distance from its serving BS, $\beta$ is the path loss exponent, $\sigma^{2}$ denotes the thermal noise at the receiver and $\zeta$ represents the combined shadowing and fading random variable (RV). More explicitly, (1) can be re-written as:

$$
\gamma= \begin{cases}P_{0} \zeta, & P_{0} r^{\beta}<P_{\max } \\ P_{\max } r^{-\beta} \zeta, & P_{0} r^{\beta} \geq P_{\max }\end{cases}
$$

The distance at which users need their maximum power to compensate path loss completely is referred to as threshold 
distance $\left(r_{t}\right)$ and can be computed as follows:

$$
r_{t}=\left(\frac{P_{\max }}{P_{0}}\right)^{1 / \beta}
$$

Users located within $r_{t}$ can compensate path loss completely while saving some proportion of their power, whereas the users located beyond $r_{t}$ transmit with their maximum power to achieve a certain rate that is less than their desired target.

Each cell is decomposed into $K$ concentric circular rings. The circular regions between two adjacent rings are characterized by uniform path loss variation (in $\mathrm{dB}$ ) and, thus, possess non-uniform width $\Delta_{k}$. Since path loss varies exponentially with distance, $\Delta_{k}$ increases from cell center to cell edge. Thus, the number of circular regions in each cell depends on the path loss exponent. The average number of users in a given ring $k$ can be computed as follows:

$$
u_{k}=\frac{U\left(r_{k}^{2}-r_{k-1}^{2}\right)}{R^{2}} \quad k=1,2, \cdots, K,
$$

where $r_{k}$ denotes the radius of ring $k$. It is important to note that $u_{k}$ can be a fraction of a number; therefore, we round off the fractional part of users in each ring. The motivation behind dividing each cell into a number of circular regions is that in each region the channel conditions of the users become relatively similar especially for large values of $K$.

The proposed approach to model ICI is detailed in the following steps:

- Derive the distribution $f_{r_{\mathrm{sel}}}(r)$ of allocating a given subcarrier to a user at a distance $r_{\text {sel }}$ from its BS.

- Derive the distribution of the distance between the allocated interfering users and the BS of the cell of interest, i.e., determine $f_{\tilde{r}_{\text {sel }}}(\tilde{r})$ using $f_{r_{\text {sel }}}(r)$ where $\tilde{r}_{\text {sel }}$ is the distance between interfering users and the BS of interest.

- Derive the distribution of the ICI $f_{X_{l}}(x)$ from the allocated user in neighboring cell $l$ to the BS of interest. Since the allocated interfering user can transmit with different power levels depending on the distance from its own serving BS, the incurred interference can be modeled as

$$
X_{l}=\left\{\begin{array}{lll}
P_{0} r^{\beta} \tilde{r}^{-\beta} \chi & \tilde{r} \in\left[\begin{array}{ll}
D-r_{t} & D+r_{t}
\end{array}\right], r \in\left[\begin{array}{ll}
0 & r_{t}
\end{array}\right] \\
P_{\max } \tilde{r}^{-\beta} \chi & \text { otherwise }
\end{array}\right.
$$

where $D=2 R$ and $\chi$ denotes the combined shadowing and fading component of the interference statistics.

- Derive the MGF of the cumulative interference $Y=$ $\sum_{l=1}^{L} X_{l}$ caused by the allocated interfering users in all neighboring cells.

\section{PMF OF ALLOCATED USER LOCATIONS}

In this section, we derive the discrete distribution of the distance of the allocated users in a given cell, i.e., the probability mass function (PMF) of $r_{\text {sel }}$. The derivation is divided into two steps explained as follows:

Step 1 (Selecting the user with the highest SNR in ring $k$ ): Since each circular region has uniform path loss variation, the users within a ring $k$ are assumed to be subject to approximately the same path loss. Thus, selecting a user in a ring $k$ is equivalent to selecting the user with maximum channel gain among all the users in ring $k$, i.e.,

$$
\zeta_{k}=\max \left\{\zeta_{1}, \zeta_{2}, \cdots, \zeta_{i}, \cdots, \zeta_{u_{k}}\right\}
$$

For simplicity, we consider independent and identically distributed (i.i.d.) channel gains of all users. Therefore, for any ring $k$, the $\mathrm{CDF}$ and PDF of the maximum channel gain $\zeta_{k}$

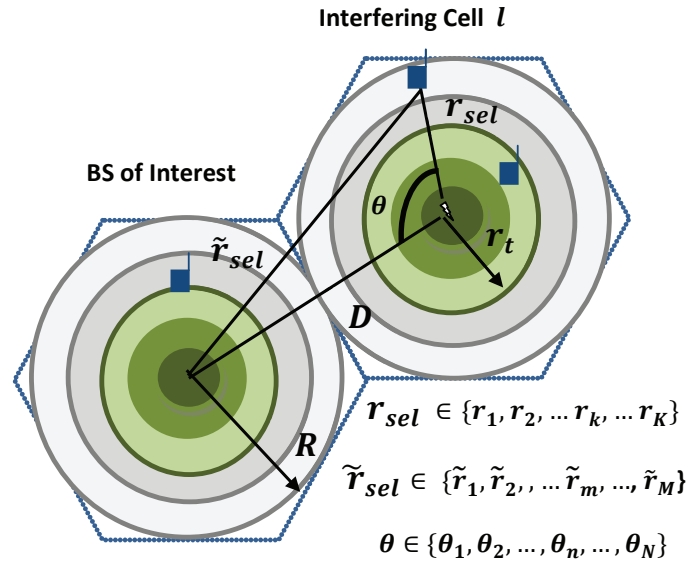

Fig. 1. Geometrical illustration of the cell decomposition into multiple rings

can be written as follows, respectively:

$$
\begin{gathered}
F_{\zeta_{k}}\left(\zeta_{k}\right)=\prod_{i=1}^{u_{k}} F_{\zeta_{i}}\left(\zeta_{k}\right)=\left(F_{\zeta}\left(\zeta_{k}\right)\right)^{u_{k}} \\
f_{\zeta_{k}}\left(\zeta_{k}\right)=\sum_{j=1}^{u_{k}} f_{\zeta_{j}}\left(\zeta_{k}\right) \prod_{i=1, i \neq j}^{u_{k}} F_{\zeta_{i}}\left(\zeta_{k}\right)=u_{k} f_{\zeta}\left(\zeta_{k}\right)\left(F_{\zeta}\left(\zeta_{k}\right)\right)^{u_{k}-1}
\end{gathered}
$$

Considering the model in (2), we split the analysis into two regions, namely the region within the threshold distance and the region beyond the threshold distance. After performing the $\mathrm{RV}$ transformation, we can write the $\mathrm{CDF}$ of the selected user SNR in each ring $k\left(\gamma_{k}\right)$ as follows:

$$
F_{\gamma_{k}}\left(\gamma_{k}\right)= \begin{cases}\left(F_{\zeta}\left(\frac{\gamma_{k}}{P_{0}}\right)\right)^{u_{k}}, & r_{k}<r_{t} \\ \left(F_{\zeta}\left(\gamma_{k} r_{k}^{\beta}\right)\right)^{u_{k}}, & r_{k} \geq r_{t}\end{cases}
$$

Step 2 (Selecting the ring $k$ with maximum SNR from the $K$ rings): In this step, we compute the probability of selecting $k^{\text {th }}$ ring among all other rings. It is important to note that this is equivalent to selecting the ring $k$ which possesses the user with the highest SNR among all rings. Thus, conditioning on $\gamma_{k}$, the PMF of $r_{\text {sel }}$ can be written as follows:

$$
P\left(r_{\text {sel }}=r_{k} \mid \gamma_{k}\right)=\prod_{\substack{i=1 \\ i \neq k}}^{K} F_{\gamma_{i}}\left(\gamma_{k}\right)
$$

By averaging over $\gamma_{k}$, the final expression for the PMF of $r_{\text {sel }}$ can be written as follows:

$$
P\left(r_{\text {sel }}=r_{k}\right)=\int_{0}^{\infty} P\left(r_{\text {sel }}=r_{k} \mid \gamma_{k}\right) f_{\gamma_{k}}\left(\gamma_{k}\right) d \gamma_{k}
$$

The result in (11) can be evaluated accurately using standard mathematical software packages such as MAPLE and MATHEMATICA and is valid for any composite fading statistics.

Note that $P\left(r_{\mathrm{sel}}=r_{k}\right)$ in (11) is the marginal PMF of $P\left(r_{\text {sel }}=r_{k}, \theta=\theta_{n}\right)$ where $\theta$ is the angular position of the allocated user and is distributed uniformly from 0 to $2 \pi$. Although the RV $\theta$ possesses continuous distribution, we discretize it in order to reduce complexity. Thus, discretizing $\theta$ into $N$ uniform angular intervals, $P\left(\theta=\theta_{n}\right)$ is $1 / N$, where $\theta_{n}$ denotes any discrete value that the RV $\theta$ can take. Since 
$r_{\mathrm{sel}}$ and $\theta$ are independent, their joint PMF can be written as:

$$
P\left(r_{\mathrm{sel}}=r_{k}, \theta=\theta_{n}\right)=\frac{P\left(r_{\mathrm{sel}}=r_{k}\right)}{N}
$$

\section{Distribution And MGF of the ICI}

In this section, firstly we find the distribution of the distance of the users allocated in the interfering cell $l$ to the BS of interest, i.e., $f_{\tilde{r}_{s e l}}(\tilde{r})$. Based on the derived expression we derive the distribution of ICI from $l^{\text {th }}$ interfering cell, i.e., $f_{X_{l}}(x)$ and the MGF of the cumulative ICI $Y$.

\section{A. Distribution of the allocated interfering user locations}

Since, each cell is assumed to have identical conditions, $f_{\tilde{r}_{\mathrm{sel}}}(\tilde{r})$ remains the same for all interfering cells and we will not use subscript $l$ any further to simplify notations. Using the cosine law, we can write:

$$
\tilde{r}_{\mathrm{sel}}^{2}=r_{\mathrm{sel}}^{2}+D^{2}-2 r_{\mathrm{sel}} D \cos \theta
$$

where $\tilde{r}_{\text {sel }}$ is the distance of the selected interfering user in cell $l$ from the BS of interest, $r_{\text {sel }}$ is the distance of the selected interfering user from its own BS, i.e., (BS $l$ ), $\theta \in\{0,2 \pi\}$.

In order to determine the PMF of $\tilde{r}_{\text {sel }}$ where $\tilde{r}_{\text {sel }} \in\{D-$ $R, D+R\}$, first of all we define $\tilde{r}_{n, k}$ for given $\theta_{n}$ and $r_{k}$ using (13) as follows:

$$
\tilde{r}_{n, k}^{2}=r_{k}^{2}+D^{2}-2 r_{k} D \cos \theta_{n} \quad \forall r_{k}, \forall \theta_{n}
$$

Clearly $\tilde{r}_{n, k}$ are the points at which $P\left(\tilde{r}_{\text {sel }}=\tilde{r}_{n, k}\right)$ can be defined using (11) as

$$
P\left(\tilde{r}_{\mathrm{sel}}=\tilde{r}_{n, k}\right)=\frac{P\left(r_{\mathrm{sel}}=r_{k}\right)}{N}
$$

The two dimensional (2-D) data set of $\tilde{r}_{\text {sel }}$, at which $P\left(\tilde{r}_{\text {sel }}=\right.$ $\left.\tilde{r}_{n, k}\right)$ is defined, can then be grouped into $M$ arcs of any arbitrary width $\Delta$. This can be done by dividing the distance between $D-R$ and $D+R$ into $M$ equal arcs of width $\Delta$ and mapping $\tilde{r}_{n, k}$ accordingly. Clearly, by adding all the probabilities for which $\tilde{r}_{\text {sel }}$ lies on the $m^{\text {th }}$ arc we get the probability of $\tilde{r}_{\mathrm{sel}}=\tilde{r}_{m}$ :

$$
P\left(\tilde{r}_{\mathrm{sel}}=\tilde{r}_{m}\right)=\sum_{\tilde{r}_{n, k} \in\left[\tilde{r}_{m}-\frac{\Delta}{2}, \tilde{r}_{m}+\frac{\Delta}{2}\right]} P\left(\tilde{r}_{\mathrm{sel}}=\tilde{r}_{n, k}\right)
$$

where $\tilde{r}_{m}$ denotes any discrete value that the RV $\tilde{r}_{\text {sel }}$ can take.

\section{B. Distribution of the ICI from one interfering cell}

Since the interfering users can transmit with different power levels depending on their distance from their serving BS, the interference $X$ can be categorized into two regions mentioned as follows:

$$
X=\left\{\begin{array}{lll}
P_{0} r_{k}^{\beta} \tilde{r}_{m}^{-\beta} \chi & \tilde{r}_{m} \in\left[\begin{array}{ll}
D-r_{t} & D+r_{t}
\end{array}\right], r_{k} \in\left[\begin{array}{ll}
0 & r_{t}
\end{array}\right] \\
P_{\max } \tilde{r}_{m}^{-\beta} \chi & \text { otherwise }
\end{array}\right.
$$

where $\chi$ denotes the interference channel statistics and for a given interferer at a distance $\tilde{r}_{m}$ from the BS of interest, the interferer distance from its own serving BS can be mapped as $r_{k}=\left|D-\tilde{r}_{m}\right|$. The PDF of $X$ conditioned on $\tilde{r}_{m}$ can be determined by RV transformation as follows:

$$
f_{X \mid \tilde{r}_{m}}(x)= \begin{cases}\frac{\tilde{r}_{m}^{\beta} f_{\chi}\left(\frac{x}{P_{0}}\left|D-\tilde{r}_{m}\right|^{-\beta} \tilde{r}_{m}^{\beta}\right)}{P_{0}\left|D-\tilde{r}_{m}\right|^{\beta}} & \tilde{r}_{m} \in\left[D-r_{t} D+r_{t}\right] \\ \frac{\tilde{r}_{m}^{\beta}}{P_{\max }} f_{\chi}\left(\frac{x}{P_{\max }} \tilde{r}_{m}^{\beta}\right) & \text { otherwise }\end{cases}
$$

Simply averaging over $\tilde{r}_{m}$ and letting $A=\frac{1}{P_{0}} \tilde{r}_{m}^{\beta}\left|D-\tilde{r}_{m}\right|^{-\beta}$ and $B=\frac{1}{P_{\max }} \tilde{r}_{m}^{\beta}$ we can write the distribution of interference, i.e., $f_{X}(x)$ as shown below:

$f_{X}(x)= \begin{cases}\sum_{\tilde{r}_{m}} A P\left(\tilde{r}_{\text {sel }}=\tilde{r}_{m}\right) f_{\chi}(A x) & \tilde{r}_{m} \in\left[\begin{array}{ll}D-r_{t} & D+r_{t}\end{array}\right] \\ \sum_{\tilde{r}_{m}} B P\left(\tilde{r}_{\mathrm{sel}}=\tilde{r}_{m}\right) f_{\chi}(B x) & \text { otherwise }\end{cases}$

Finally, $f_{X}(x)$ can be written explicitly as follows:

$$
\begin{array}{r}
f_{X}(x)=\sum_{\tilde{r}_{m} \in\left[D-r_{t}\right.} A P\left(\tilde{r}_{\mathrm{sel}}=\tilde{r}_{m}\right) f_{\chi}(A x)+ \\
\sum_{\tilde{r}_{m} \notin\left[D-r_{t}\right]} B P\left(\tilde{r}_{\mathrm{sel}}=\tilde{r}_{m}\right) f_{\chi}(B x)
\end{array}
$$

\section{MGF of the cumulative ICI}

Computing the distribution of the cumulative ICI $Y$ requires the convolution of the PDF of $L$ RVs $X_{l}, \forall l=1,2, \cdots L$, which is a tedious task for many practical scenarios. To avoid the convolution operations, we utilize an MGF based approach. Since the scheduling scheme is considered to be identical in all cells, the interferers are i.i.d. and therefore the MGF of the cumulative interference $Y$ can be written as follows:

$$
\mathcal{M}_{Y}(t)=\prod_{l=1}^{L} \mathcal{M}_{X_{l}}(t)=\left(\mathcal{M}_{X}(t)\right)^{L}=\left(\mathbb{E}\left[e^{t x}\right]\right)^{L}
$$

Looking at the structure of (19), we can derive MGF of any composite fading model as $\mathcal{M}_{X}(t)=\int_{0}^{\infty} e^{t x} f_{X}(x) d x$ The expression applies to any kind of composite fading models. Due to space limitations we will study only the MGF of the Gamma composite fading case, i.e., we consider a scenario in which shadowing and fading statistics are modeled by a Gamma and Nakagami distribution (also referred as Generalized-K [10]), respectively. Recently, in [10] an accurate approximation of the Generalized-K RV using moment matching method has been proposed to increase its analytical tractability, i.e., the Generalized-K distribution can be approximated by a simple Gamma distribution [10]. Therefore in this case $\mathcal{M}_{X}(t)$ ) can be derived as follows:

$$
\begin{array}{r}
\mathcal{M}_{X}(t)=\sum_{\tilde{r}_{m} \in\left[D-r_{t}\right.} \sum_{\left.D+r_{t}\right]} \frac{A^{m_{s}} P\left(\tilde{r}_{\mathrm{sel}}=\tilde{r}_{m}\right)}{\left(A-m_{c} t\right)^{m_{s}}}+ \\
\sum_{\tilde{r}_{m} \notin\left[D-r_{t}\right.} \frac{B^{m_{s}} P\left(\tilde{r}_{\mathrm{sel}}=\tilde{r}_{m}\right)}{\left(B+r_{t}\right]}
\end{array}
$$

Finally $\mathcal{M}_{Y}(t)$ can be given simply using (21).

\section{Evaluation of Network Performance Metrics}

In this section, we will utilize the derived expressions to evaluate the network ergodic capacity, average fairness, and average power preservation per user. The evaluation of outage probability is skipped due to space limitations, however, the readers can refer to [7] for details.

\section{A. Evaluation of Network Ergodic Capacity}

Using the lemma derived in [11], the following expression is valid for interference limited systems:

$$
\mathbb{E}\left[\ln \left(1+\frac{X_{0}}{\sum_{l=1}^{L} X_{l}}\right)\right]=\int_{0}^{\infty} \frac{\mathcal{M}_{Y}(t)-\mathcal{M}_{X_{0}, Y}(t)}{t} d t
$$


where, $\mathcal{M}_{Y}(t)=\mathbb{E}\left[e^{-t \sum_{l=1}^{L} X_{l}}\right]$ is the MGF of the cumulative interference and $\mathcal{M}_{X_{0}, Y}(t)=\mathbb{E}\left[e^{-t\left(X_{0}+\sum_{l=1}^{L} X_{l}\right)}\right]=$ $\mathbb{E}\left[e^{-t\left(X_{0}+Y\right)}\right]$ is the joint MGF of the corresponding signal power of the scheduled user $X_{0}$ and cumulative interference $Y$. Since $X_{0}$ and $Y$ are independent, $\mathcal{M}_{X_{0}, Y}(t)=$ $\mathcal{M}_{X_{0}}(t) \mathcal{M}_{Y}(t)$. The expression for $\mathcal{M}_{X_{0}}(t)$ can be given as:

$$
\mathcal{M}_{X_{0}}(t)=\int_{0}^{\infty} e^{t x_{0}} f_{X_{0}}\left(x_{0}\right) d x_{0}=\int_{0}^{\infty} t e^{t x_{0}} F_{X_{0}}\left(x_{0}\right) d x_{0}
$$

where $F_{X_{0}}\left(x_{0}\right)=\prod_{i=1}^{K} F_{\gamma_{k}}\left(x_{0}\right), f_{X_{0}}\left(x_{0}\right)=\frac{\partial}{\partial x_{0}} F_{X_{0}}\left(x_{0}\right)$. Closed form expressions are also available for $\mathcal{M}_{X_{0}}(t)$ in the literature [12].

\section{B. Evaluation of Average Fairness and Power Preservation}

To measure the degree of fairness among users, we follow the notion developed in [13]. The average fairness in a network with $U$ users is defined as $\mathcal{F}=-\sum_{i=1}^{U} p_{i} \frac{\log p_{i}}{\log U}$ where $p_{i}$ is the proportion of resources allocated to a user $i$ or the access probability of user $i$. A system is strictly fair if each user has equal probability to access the channel and in such case the average fairness becomes one. The average fairness can be easily computed using our derived results as:

$$
\mathcal{F}=-\sum_{k=1}^{K} P\left(r_{\text {sel }}=r_{k}\right) \frac{\log P\left(r_{\text {sel }}=r_{k}\right)-\log u_{k}}{\log U}
$$

where $P\left(r_{\text {sel }}=r_{k}\right)$ is given by (11). Moreover, the average power savings per subcarrier can also be calculated as follows:

$$
\bar{P}=\sum_{r_{k}=r_{1}}^{r_{t}} P\left(r_{\text {sel }}=r_{k}\right)\left(P_{\max }-P_{0} r_{k}^{\beta}\right)
$$

where $r_{t}$ denotes the threshold distance.

\section{RESUlts AND ANALYSis}

In this section, we aim to validate the accuracy of the derived expressions through Monte-Carlo simulations. The results are presented for Gamma composite fading, i.e., $\zeta \sim$ $\operatorname{Gamma}(1,1), \chi \sim \operatorname{Gamma}(3 / 2,2 / 3)$. Firstly we will provide a brief overview of the Monte-Carlo simulation setup.

1) Generate $U$ uniformly distributed users per cell. Each user has an instantaneous SNR given by (1). Select a user with maximum instantaneous SNR. Store the distance of the selected user, i.e., $r_{\text {sel }}$ from the serving BS.

2) Compute the distance of the selected user from the BS of interest, i.e., $\tilde{r}_{\text {sel }}$ using cosine law and finally generate the interference using (5).

3) Repeat all steps for a large number of iterations. Generate histogram for the discrete RV $r_{\text {sel }}$ with non-uniform bin widths and another histogram for the discrete RV $\tilde{r}_{\text {sel }}$ with any arbitrary bin width equal to $\Delta$. To compute capacity, we consider

$$
\Delta= \begin{cases}r_{t} / 5 & \tilde{r} \in\left[\begin{array}{ll}
2 R-5 r_{t} / 6 & 2 R+5 r_{t} / 6
\end{array}\right] \\
\left(R-r_{t}\right) / 8 & \text { otherwise }\end{cases}
$$

In Fig. 2 the impact of the maximum transmit power limit is shown on the PMF of allocated user locations. The obtained PMF results fit well with exhaustive Monte-Carlo simulations. Since the users located within the threshold region $r_{t}$ can compensate their distance based path loss, each user has on average equal probability of allocation within $r_{t}$. The increasing trend of PMF within $r_{t}$ is therefore simply due to an increase in the number of users in each ring from cell center to the cell edge. It is important to note that the users located beyond $r_{t}$ transmit with their maximum power as they cannot compensate path loss. These users are therefore scheduled based on their relative channel gains which prioritizes close users over the far users and hence causes rapid decay of allocation probability beyond $r_{t}$. In greedy scheduling [7], the cell center users have higher priority to be allocated over the cell edge users. On the other side, round robin scheduling provides equal probability of allocation to each user, hence high probability of allocation near the cell edge due to the large area and large number of users at the cell-edge. By observing the result, it can be concluded easily that greedy scheduling with power control (PC) follows the trend of round robin within $r_{t}$ whereas the trend of greedy scheduling beyond $r_{t}$. The performance of greedy scheduling with PC is therefore expected to lie in between the two extremes.

Two different transmit power limits are also studied in Fig. 2 which yields two threshold distances, i.e., $r_{t}=400 \mathrm{~m}$ and $r_{t}=260 \mathrm{~m}$, respectively. It can be observed that the greater the maximum transmit power, the greater is the threshold distance and more users located farther from the BS become capable to compensate path loss which increases fairness and in turn the incurred ICI. The slight mismatch in the simulations and analysis demonstrates the impact of discretization which is dominant for channel based scheduling beyond $r_{t}$. However, this error can be reduced by increasing the number of rings. In Fig. 3, the PMF of the distance of allocated interfering users is plotted. The slight descend in the middle is due to the rounding of users near the cell center. Even though, the higher transmit power leads to high probability of allocating the cell edge users and large ICI, the aggregate amount of interference incurred is still lower compared to the greedy scheduling with no PC, i.e., in which all users transmit with their maximum power. This reduction in the amount of ICI is quantified and discussed in more detail in Fig. 4.

The CDF of the ICI for different transmit power budgets and different path loss exponents for greedy scheduling with and without PC is plotted in Fig. 4. High values of path loss exponents causes rapid signal degradation, hence, reduces ICI. Moreover, it can be observed clearly that with low user transmit powers, there is a significant reduction in ICI compared to the high transmission powers. It is further interesting to note that the performance of greedy scheduling with PC always remain better than the greedy scheme in terms of incurred ICI, average fairness (see Fig. 5part(a)) and average power consumption of the users. The top figure in Fig. 5 quantifies the average fairness of the greedy with and without PC and round robin schedulers. With the increase of transmit powers, far users can also adapt their power which increases the average fairness among users. For high user transmit powers, the greedy scheduling with PC achieves the fairness of round robin scheme as is also evident from Fig. 2.

The bottom figure in Fig. 5 demonstrate the network capacity of interference limited systems (i.e., thermal noise is neglected). Without PC, the performance of greedy and round robin scheduling remains independent of the transmit power as the factor of $P_{\max }$ cancels out in the capacity calculation. However, since the greedy scheduling with PC have less ICI then the greedy scheduler, the network capacity is expected to increase which is not the case as the corresponding user transmit powers are also lowered along with the interfering powers. The main reason of the capacity degradation with the increase in transmit power budget is that the greater transmission power more users can compensate path loss which reduces the number of users transmitting with their maximum powers. This phenomena on one hand increase 


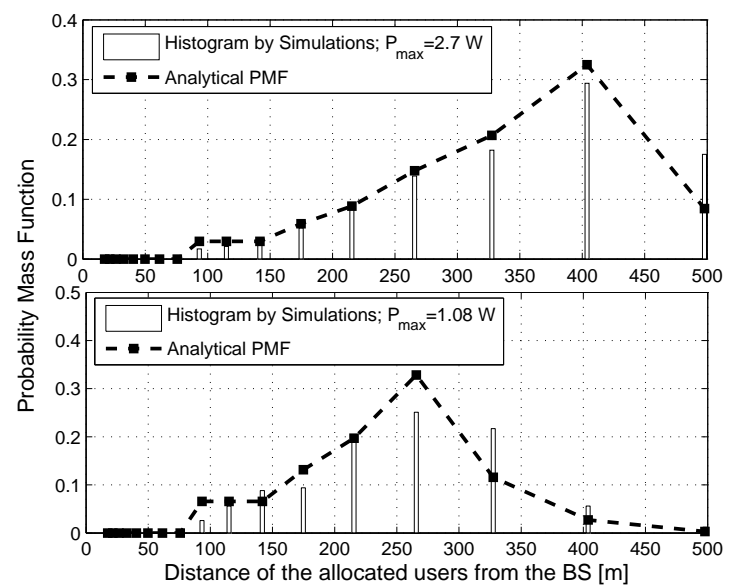

Fig. 2. PMF of the distance of allocated users from their serving BS in a given cell (i.e., PMF of $r_{\mathrm{sel}}$ ) for greedy scheduler with power adaptation, $R=500 \mathrm{~m}, \beta=2.2, U=50, P_{0}=-23 \mathrm{dBm}$.

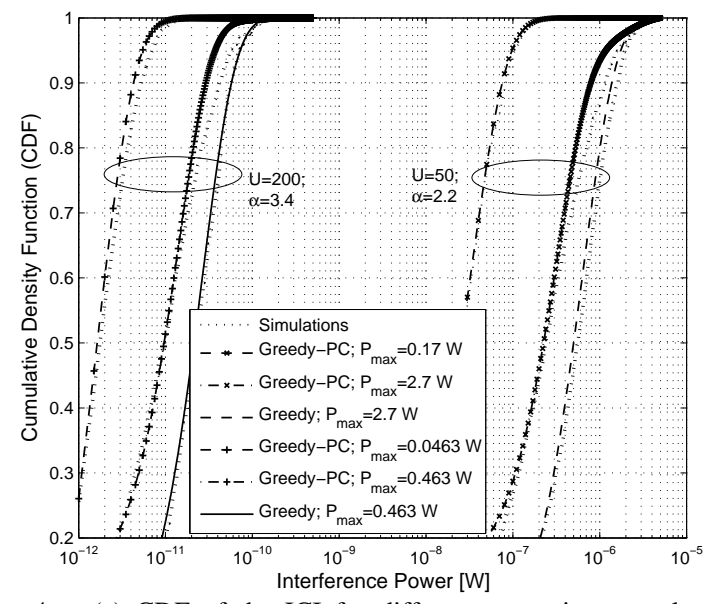

Fig. 4. (a) CDF of the ICI for different transmit power levels and path loss exponents considering greedy scheduling with and without power control (PC), $P_{0}=-23 \mathrm{dBm}, R=500 \mathrm{~m}$.

average power savings whereas on the other hand degrades overall system capacity.

\section{CONCLUSIONS}

We presented an approach to model uplink ICI considering the impact of greedy scheduler and power adaptation based on channel conditions. The presented approach applies to a wide range of channel models. The derived expressions are shown to be useful in evaluating important network performance metrics without the need of time consuming Monte-Carlo simulations.

\section{REFERENCES}

[1] Vodafone Chair, "CoolCellular - Energy Efficient Network Architectures and Transmission Methods," avaiable at http://www.vodafonechair. com/research/projects cool cellular.html.

[2] G.W. Miao, N. Himayat, Y. Li, and A. Swami, "Cross-layer optimization for energy-efficient wireless communications: A survey," Wireless Commun. and Mobile Computing, vol. 9, no. 4, pp. 529-542, 2009.

[3] K.W. Sung, H. Haas, and S. McLaughlin, "A semianalytical PDF of downlink SINR for femtocell networks," EURASIP Journal on Wireless Communications and Networking, Jan. 2010.

[4] S. Plass, X.G. Doukopoulos, and R. Legouable, "Investigations on linklevel inter-cell interference in OFDMA systems," IEEE Symposium on Communications and Vehicular Technology, pp. 49-52, Liege, Nov. 2006.

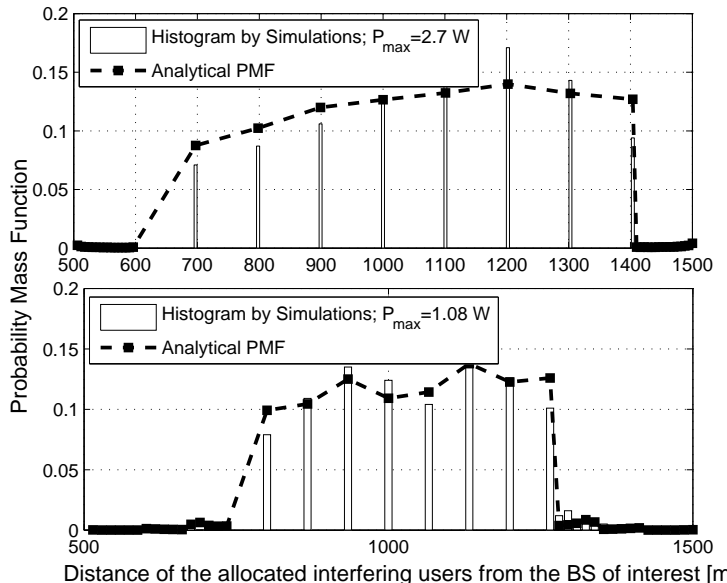

Fig. 3. PMF of the distance at which the users in the interfering cells are allocated (i.e., PMF of $\tilde{r}_{\text {sel }}$ ) for greedy scheduler with power adaptation, $R=500 \mathrm{~m}, \beta=2.2, U=50, P_{0}=-23 \mathrm{dBm}$.

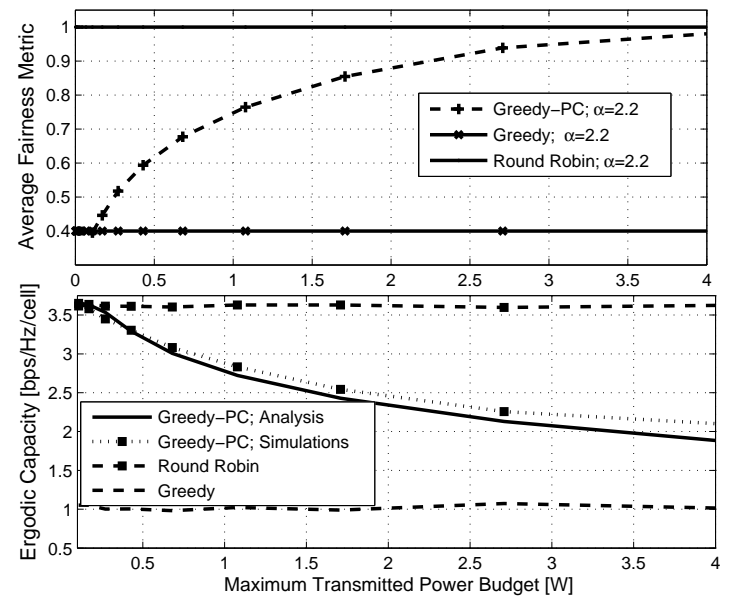

Fig. 5. (a) Average fairness among users considering greedy scheduler with and without power control (PC) and round robin scheduler without PC (b) Ergodic capacity considering greedy scheduler with and without $\mathrm{PC}$ and round robin scheduler without $\mathrm{PC}, U=50, P_{0}=-23 \mathrm{dBm}, R=500 \mathrm{~m}, \beta=2.2$.

[5] S. Elayoubi, B. Haddada, and B. Fourestie, "Performance evaluation of frequency planning schemes in OFDMA based networks," IEEE Transactions on Wireless Communications, vol. 7, no. 5, pp. 1623-1633, May 2008.

[6] R. Kwan and C. Leung, "On collision probabilities in frequency-domain scheduling for LTE cellular networks," IEEE Communication Letters, vol. 15, no. 9, pp. 965-967, Sep. 2011.

[7] H. Tabassum, F. Yilmaz, Z. Dawy, and M-S. Alouini , "On the statistics of uplink inter-cell interference with greedy resource allocation," to appear in IEEE International Symposium on Wireless Communication Systems, ISWCS, Aachen, Germany, November, 2011.

[8] A. Simonsson and A. Furuskar, "Uplink Power Control in LTE Overview and Performance, Subtitle: Principles and Benefits of Utilizing rather than Compensating for SINR Variations," IEEE Vehicular Technology Conference, (VTC'08).

[9] Y. Zhao, "Standardization of mobile phone positioning for 3G systems," IEEE Communications Magazine, vol. 40, no. 7, pp. 108-116, 2002.

[10] S. Al-Ahmadi and H. Yanikomeroglu, "On the approximation of the generalized-K PDF by a Gamma PDF using the moment matching method," Wireless Communications and Networking Conference, (WCNC'09), pp. 1-6, Budapest, Hungary, Apr. 2009.

[11] K.A. Hamdi, "A useful lemma for capacity analysis of fading interference channels," IEEE Transactions on Communications, vol. 58, no. 2, pp. 411-416, Feb. 2010.

[12] J. M. Romero-Jerez, and A. J. Goldsmith, "Performance of multichannel reception with transmit antenna selection in arbitrarily distributed Nagakami fading channels," IEEE Transactions on Wireless Communications, vol. 8, no. 4, pp. 2006-2013, 2009.

[13] I. M. Kostic, "Analytical approach to performance analysis for channel subject to shadowing and fading," IEE Proceedings Commmunication, vol. 152, no. 6, pp. 821-827, Dec. 2005. 